\title{
A study of magnetically-supported dc discharge in cylindrical and inverted cylindrical configuration
}

\author{
O. Bilyk $\S$, P. Kudrna, M. Holík, A. Marek, M. Tichý \\ Charles University in Prague, Faculty of Mathematics and Physics, \\ V Holešovičkách 2, 18000 Prague 8, Czech Republic
}

\begin{abstract}
.
We have investigated apparently stochastic fluctuations of magnetically-supported dc discharge in cylindrical coaxial configuration. In the system the electric field had radial direction while the magnetic field was applied axially. The discharge vessel length was 12 centimetres. Working gas was typically argon at pressure of several $\mathrm{Pa}$, magnetic field 10-50 mT.

The contribution describes experimental results - frequency and phase analysis of the instabilities, which we detected in our experimental system in both the conventional and inverted magnetron configurations.

We bring also 2-D PIC model of the dc discharge under conditions, which can be achieved in the experimental apparatus. The PIC model should answer questions, which originated from the experimental study of the plasma parameters - presented e.g. in [1].
\end{abstract}

$\S$ To whom correspondence should be addressed (bilyk@mbox.troja.mff.cuni.cz) 


\section{Introduction}

Magnetically-supported dc discharges in cylindrical symmetry (cylindrical magnetrons) are used for deposition of high-temperature superconducting materials, see e.g. [2], or materials with special dielectric characteristics, see e.g. [3]. Understanding the behavior of the dc discharge in such configuration is therefore essential condition for the technological progress in this branch. The magnetron systems with cylindrical symmetry are relatively simple and thus the plasma processes in them can be simulated comparatively easily by computer models. Measurements of plasma parameters in cylindrical magnetron can thus be compared with theoretical predictions and/or calculations used for their verification.

In this contribution results of the potential fluctuations measurements and 2-D PIC modelling are brought. As we presented in former papers, e.g. [1], by the study of axial and radial dependence of the plasma parameters, the axial inhomogeneities were observed, which couldn't be reproduced by the 1-D PIC model. The 2-D model was then used to try to explain the presence of the local minimum of the plasma density in the middle of the discharge vessel. Further research has been done in this direction and we bring new results of the calculations. In connection with the differences between computer models and the experimental results, importance is often attached to the plasma fluctuations. To answer the question how big role play the fluctuations in our system, we measured potential fluctuations with two parallel probes and evaluated frequency spectra as well as a correlation of the signals from the both probes to check presence of coherent modes 4] in the discharge.

\section{Experimental}

\subsection{Experimental setup}

The cylindrical magnetron in the so-called post discharge configuration consists of cylindrical cathode mounted coaxially inside the anode. The discharge volume is axially limited by means of two disc-shaped limiters, which are connected to the cathode potential. Our device is schematically depicted in Fig. 11 the electrodes configuration is shown in Fig. 2. The diameters of the cathode and anode are $10 \mathrm{~mm}$ and $60 \mathrm{~mm}$, respectively. The length of the discharge volume is $120 \mathrm{~mm}$. The homogeneous magnetic field is created by two coils and is in parallel with the common axis of the system. To prevent overheating the coils and cathode, the system is cooled by water.

The apparatus is constructed as high vacuum. The pumping unit consists of the combination of the turbomolecular and rotary pumps. The ultimate presure is in the order of $10^{-3} \mathrm{~Pa}$. During the operation of the magnetron discharge the working gas, in this case argon, slowly flows into the system and through the valve reducing the pumping speed of the pumps leaves the system. The typical flow rate is below $1 \mathrm{sccm}$ and is adjusted by means of the MKS flow controller in order to keep the pressure in the discharge volume constant. 
The system is equipped with several diagnostic ports, see Fig. 11. One of them can be used to install a radially movable Langmuir probe. Axially movable probe can be installed using the port at the side of the vacuum vessel. For measurements of the plasma potential fluctuations the axial movable probe holder was used to enable measuring with a mean of two Langmuir probes placed as clear from Fig. 1.

\subsection{Estimation of the power spectra}

The Langmuir probes were used in floating regime, i.e. without the applied bias voltage. The floating potential signal was sampled using the digital oscilloscope (Tektronix TDS 610) and samples $h_{n}$ were transferred to the computer over the GPIB interface

$$
h_{n}=h(n . \Delta t)
$$

where $h(t)$ is the probe voltage with respect to the anode, $\Delta t$ is the sampling interval and integer $n$ ranges from 0 to the number of samples $N-1$. Then the discrete Fourier

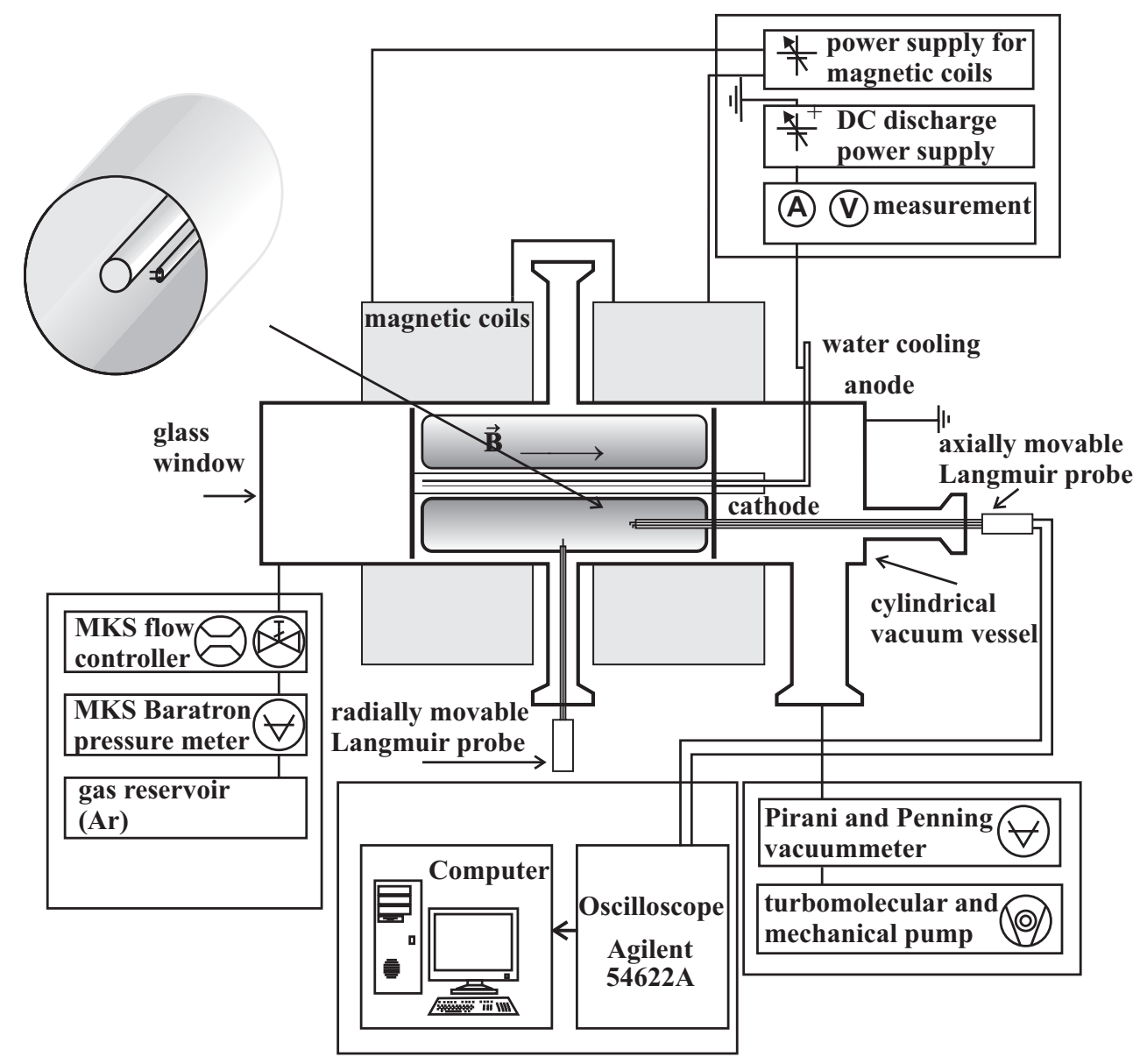

Figure 1. Experimental set-up of the cylindrical magnetron in coaxial configuration. 


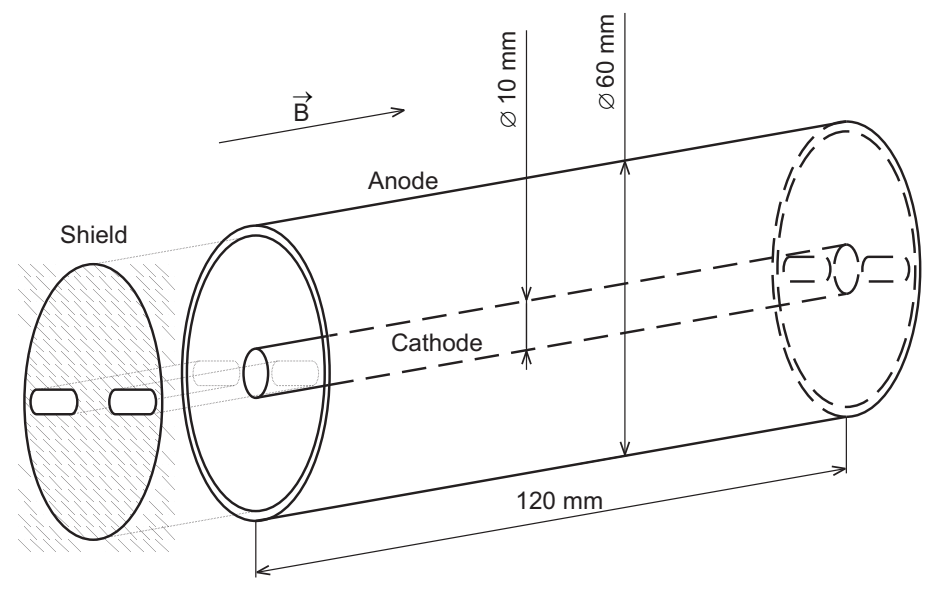

Figure 2. Geometric configuration of the magnetron electrodes - the cathode is coaxially placed in the middle of the vacuum vessel. In the inverted configuration the outer electrode serves as a grounded cathode and the anode is connected to a positive dc power supply.

transform of the sampled data was calculated by means of the FFT:

$$
H_{k}=\sum_{n=0}^{N-1} h_{n} \exp \frac{-2 \pi \mathrm{i} k n}{N} .
$$

The guess of the Fourier transform of the voltage $h(t)$ at the discrete frequencies $k . \Delta f$ is given by

$$
H(k . \Delta f)=\Delta t . H_{k},
$$

where $\Delta f=(N . \Delta t)^{-1}$. The power density is then

$$
S(f)=\frac{1}{N \cdot \Delta t}|H(f)|^{2} .
$$

In order to decrease the scatter of the calculated spectrum estimate several realizations (typically 100) were averaged into the resulting power density curve.

\subsection{Frequency vs. wave number spectra}

In order to analyze the wave behavior of the potential fluctuations of the magnetron discharge the spectra derived from the fluctuations measured simultaneously from two Langmuir probes were evaluated according to the method [4]. At first the wave number can be expressed for each frequency from the phase shift between the signals at the two probes and known distance $\Delta x$ between the probes:

$$
k(f)=\frac{\arg H^{(2)}(f)-\arg H^{(1)}(f)}{\Delta x} .
$$

Using the wave number and the average power density at two probes $\frac{1}{2}\left[S^{(1)}(f)+S^{(2)}(f)\right]$ the histogram $S(f, k)$ can be built. 


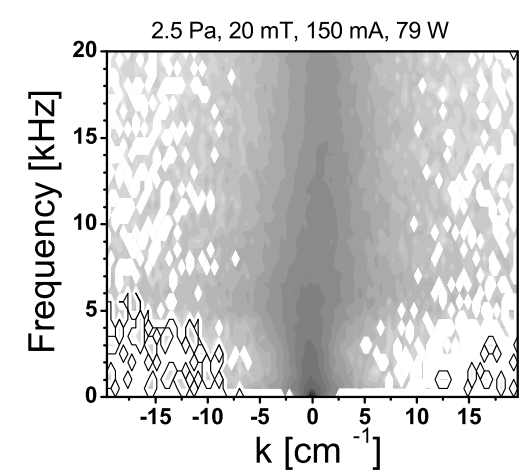

(a)

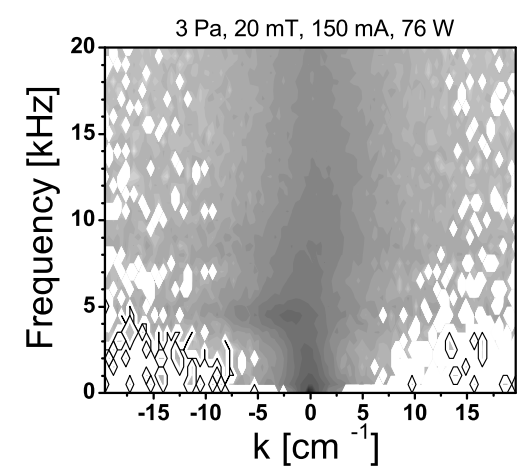

(d)

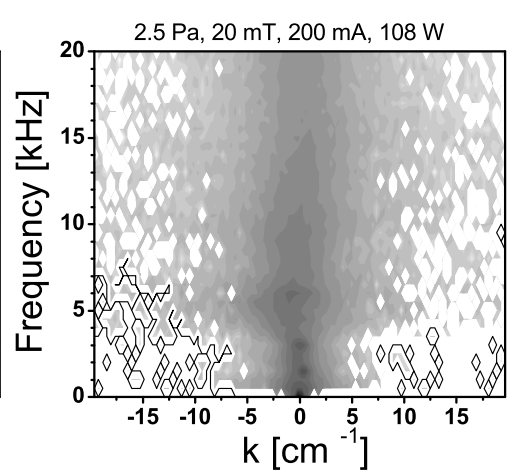

(b)

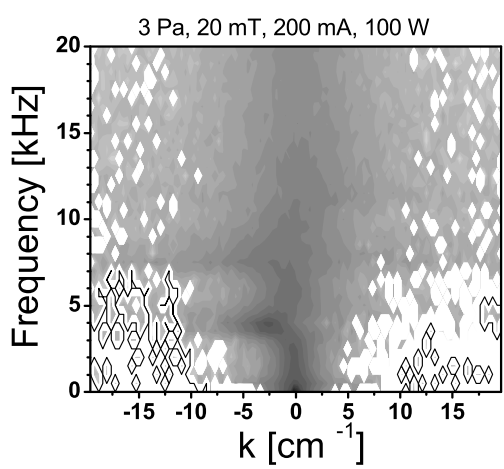

(e)

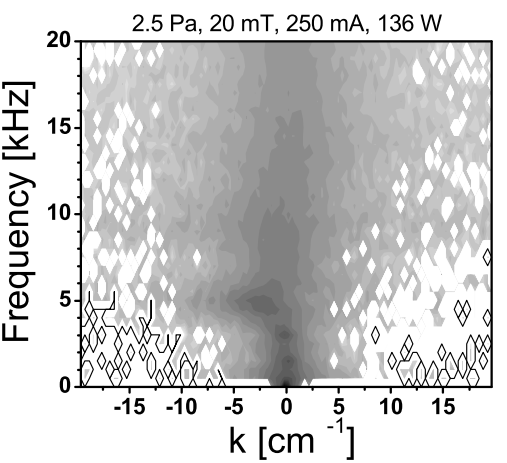

(c)

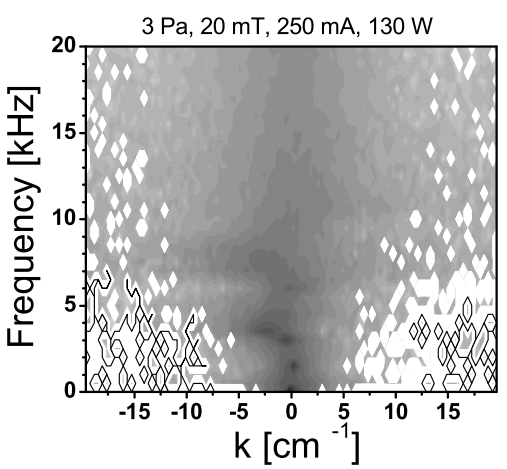

(f)

Figure 3. Frequency wave number histograms of the argon dc discharge in cylindrical magnetron in conventional arrangement at pressure $2.5 \mathrm{~Pa}$ and $3 \mathrm{~Pa}$ and at magnetic field $20 \mathrm{mT}$.

In Figure 3 there is shown an example of the frequency wave number histograms, evaluated from the simultaneous measurements with two probes at different discharge powers in the non-inverted configuration. The $z$-axis (power density axis) is showed as a tone of grey and darker areas at certain frequencies mean that the two signals are correlated with given phase shift. There can be seen certain coherent modes especially in Figures 3 through $\mathrm{f}$.

The potential fluctuations of the discharge in the inverted configuration were also investigated. However, although the measurements were done within wide range of powers and pressures, no observable coherent modes were detected in argon discharge.

\section{PIC model}

Plasma modeling techniques can be divided into two basic families with respect to the plasma description: codes using kinetic description and codes using fluid description of plasma. The technique of our interest - Particle-In-Cell (PIC) technique e.g. [5, 6, 7] - belongs among the codes that use kinetic description of plasma. In PIC technique there are solved equations of motion for all charged particles in plasma. Computation of mutual electrical forces is in the PIC approach transformed to the solving of Poisson 


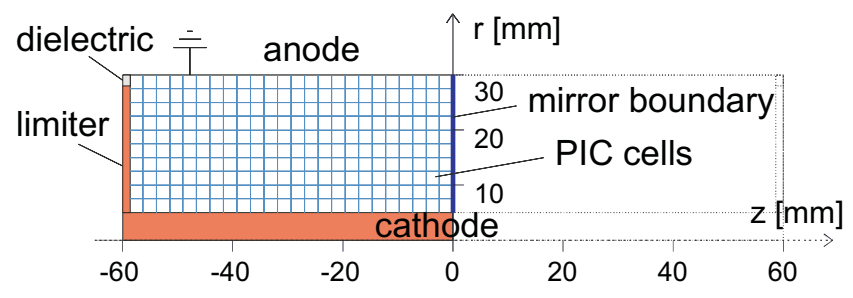

Figure 4. The electrodes configuration and the cells distribution in the discharge area.

equation in the simulated region. A great advantage of this technique is that it is self-consistent. On the other hand self-consistent approach is compensated by great computational effort (especially in 2D and 3D simulations).

The electrostatic Particle-In-Cell computational scheme consists of the following steps:

1. Division of simulated region to cells.

2. Assignment of the charge of particles to the mesh points.

3. Computing of electric field.

4. Particles move in calculated field.

5. Jump to point 2.

Although results of XOOPIC simulation presented here are in relatively good agreement with the experiment there is a problem with numerical instability of the simulation - with a growing number of computational particles during simulation. Hence, the computational model needs further improvement. A discharge current source may be implemented into the code to help to solve this problem. In future work, we hope to bring with our simulation model a more detailed study of the discharge in cylindrical magnetron and more precise comparison with experiment.

In Figure 5 there are shown the results of the PIC modeling of the dc discharge
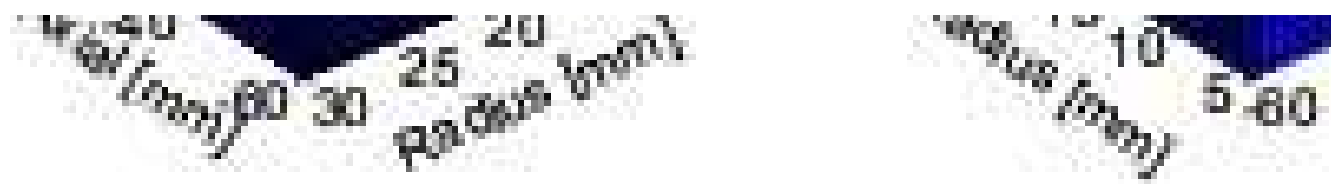

Figure 5. Results of the PIC modelling of the argon dc discharge in the cylindrical magnetron apparatus. 
in argon in the cylindrical magnetron apparatus described above. The model was done under the the following conditions: magnetic field strength $20 \mathrm{mT}$, pressure $5 \mathrm{~Pa}$, voltage $200 \mathrm{~V}$, time step $5 \mathrm{ps}$, simulation time $23.8 \mu \mathrm{s}$. Grid size was 40 in axial direction and 100 in radial direction.

\section{Conclusion}

We measured potential fluctuations and evaluated the correlation between two probes placed parallel in the discharge. The coherent modes similar to those observed in [4] were found under certain conditions in the non-inverted cylindrical magnetron configuration.

The 2-D PIC model was used to calculate plasma parameters under the same conditions, which can be established in the experimental apparatus. The results of the electron density and plasma potential were presented. The model results qualitatively agree with experimental observations [1, 8, 9, 10, further effort in modeling is still needed.

\section{Acknowledgments}

The work was financially supported by the Czech Science Foundation, Grant No. 202/03/0827, 202/04/0360, 202/03/H162, by project COST action 527.70, by the Faculty of Mathematics and Physics of Charles University in Prague, Research plan MSM 1132000002 and by EURATOM.

\section{References}

[1] M. Holík, O. Bilyk, A. Marek, P. Kudrna, J. F. Behnke, I. A. Porokhova, Yu. B. Golubovskii, M. Tichý, 2004, Contr. Plasma Phys. 44, No. 7-8, p. 613-618.

[2] G. Lengl, P. Ziemann, F. Banhart, P. Walther, 2003, Physica C 390, p. 175.

[3] M. Adam, D. Fuchs, R. Schneider, 2002, Physica C 372, p. 504.

[4] E. Martines, et.al., 2001, Phys. of Plasmas 8 6, 3042.

[5] C.K. Birdsall, A.B. Langdon, Plasma physics via computer simulation, Mc Graw-Hill 1995, AdamHilger 1991.

[6] J. Verboncoeur, A.B. Langdon and N.T. Gladd, An Object-Oriented Electromagnetic PIC Code, 1995, Comp. Phys. Comm. 87, p. 199-211.

[7] J.P. Hammel, J. Verboncoeur, DC Discharge studies using PIC-MCC, 2004, http://ptsg.eecs.berkeley.edu/jijhammel/report.pdf

[8] E. Passoth, P. Kudrna, C. Csambal, J.F. Behnke, M. Tichy, V. Helbig, 1997, J. Phys. D: Appl. Phys. 30 p. 1763-1777.

[9] I. A. Porokhova, Yu. B. Golubovskii, J. Bretagne, M. Tichy, and J.F. Behnke, 2001, Physical Review E 63.

[10] I. A. Porokhova, Yu. B. Golubovskii, M. Holik, P. Kudrna, M. Tichy, C. Wilke, and J. F. Behnke, 2003, Physical Review E 68. 